\title{
Aplicación Del Modelo De Progresión Temática Derivada En La Producción Escrita De Textos Expositivos En Inglés (Ensayo Básico)
}

\section{Logroño Becerra Mónica Alejandra \\ Rojas Castro Carmita Eulalia \\ Lara Olivo Lenin Iván}

Magister en Lingüística y Didáctica de la Enseñanza de Idiomas Extranjeros

Docente de la Escuela Superior Politécnica de Chimborazo,

Centro de Idiomas, Ecuador

\section{Guambo Yerovi Enrique Jesús}

Magister en Linguistica Aplicada al Aprendizaje del Ingles

Docente de la Escuela Superior Politécnica de Chimborazo,

Centro de Idiomas, Ecuador

\section{Doi:10.19044/esj.2018.v14n20p37～URL:http://dx.doi.org/10.19044/esj.2018.v14n20p37}

\begin{abstract}
This study focuses on the shortcomings affecting cohesion and coherence in English expository texts written by the students of sixth level of the Language Center of the Escuela Superior Politécnica de Chimborazo during October 2016 - March 2017. The derived thematic progression model to improve the expository texts production was the teaching strategy used by taking as a starting point the textual linguistics since it addresses to the analysis of the text as a product, in other words, the text as a coherent whole. In order to make this possible, it was conducted a quasi-experimental research, with a correlational level, and a quantitative-qualitative approach. Both, a pre -test and a post-test to the experimental and the control groups, were carried out, as well as a subsequent analysis of expository texts at the super structure, macro structure and microstructure levels. At the super structure level, the main parts of the text were identified. At the macro structure level, each of these parts was analyzed per unit of paragraph, and at the microstructure level, the grammatical structure of the different sentences of the paragraphs forming the text was also analyzed. This fact implied the identification and use of the theme-rheme elements, necessary to establish the order and logical sequence of ideas at local and global level of the text. Finally, in the process of writing was included the Derived Thematic Progression model, whose findings reported significant changes in the level of cohesion and coherence of the
\end{abstract}


texts, demonstrating that this strategy may become the basis to set didactic activities to improve written production

Keywords: Derived Thematic Progression Model, Cohesion, Coherence, expository texts, Written Production, English, Expository Texts

\section{Resumen}

El presente estudio aborda las limitaciones que afectan la cohesión y coherencia de los textos expositivos escritos en inglés por los estudiantes de sexto nivel del Centro de Idiomas de la Escuela Superior Politécnica de Chimborazo durante el periodo octubre 2016 - marzo 2017.Se consideró el modelo de progresión temática derivada como estrategia para la producción de textos expositivos, tomando como punto de partida la lingüística textual que estudia al texto como producto, es decir el texto visto como un todo coherente. Para ello, se aplicó una investigación de tipo cuasi-experimental, con nivel de profundidad correlacional, y enfoque investigativo cuantitativo y cualitativo. Se realizó un pre y post test al grupo experimental y de control, y subsecuentemente, un análisis de textos expositivos a nivel de súper estructura, macro estructura y microestructura. A nivel de súper estructura se identificó las partes del texto, a nivel de macro estructura se analizó cada una de dichas partes por unidad de párrafo, y a nivel de microestructura se analizó la estructura gramatical de las diferentes oraciones de los párrafos del texto. Este hecho implicó la identificación y uso de los elementos tema-rema, necesarios para establecer el orden y secuencia lógica de las ideas a nivel local y global del texto. Finalmente, en el proceso de escritura se incorporó el modelo de Progresión Temática Derivada, cuyos hallazgos reportaron cambios significativos en el nivel de cohesión y coherencia demostrando que esta estrategia puede constituir la base para determinar un conjunto de actividades didácticas encaminadas a la mejora de la producción escrita.

Palabras Clave: Modelo de Progresión Temática Derivada, Cohesión, Coherencia, Producción escrita, Inglés, Textos Expositivos.

\section{INTRODUCCIÓN}

En el proceso de enseñanza-aprendizaje de inglés como lengua extranjera, la escritura académica es uno de los aspectos que demanda constante práctica y disciplina, por esta razón, existe un creciente interés por desarrollar la competencia escrita. La investigación educativa en el ámbito de las lenguas extranjeras conlleva a reformular estrategias que contribuyan al desempeño de los/las estudiantes en la escritura de textos

Ping (2007), en su estudio "Developing the Message: Thematic Progression and Student Writing" efectuado en Singapur, comprobó el efecto 
positivo en la producción escrita de los/las estudiantes luego de diferenciar los componentes tema-rema a través de la aplicación de los modelos de progresión temática lineal y paralela, lo que a su vez contribuyó a la diferenciación entre clausula principal y clausula secundaria.

Tomando en cuenta el efecto positivo de la progresión temática, se pretende que los/las estudiantes logren asociar sus ideas de manera lógica, pues el seguir un patrón informacional facilitará a los/las estudiantes asimilar la forma en la que están transmitiendo sus ideas, a la vez que podrán reflexionar acerca de su aprendizaje y sus concepciones acerca de la realidad, pueden ser interpretadas de mejor manera.

El analizar la producción escrita desde este punto de vista no significa que exista un determinado esquema para cada tipo de texto, sin embargo, cabe mencionar que existen patrones predominantes en los diferentes tipos de texto.

Thomas Hawes (2014), en su estudio "Thematic progression in the writing of students and professionals" demostró que los/las estudiantes y profesionales en el área de periodismo, no nativo hablantes del idioma inglés; incrementaron los niveles de cohesión y coherencia de sus ensayos argumentativos y expositivos después de haber experimentado con tres diferentes modelos de progresión temática para lo cual se analizaron dos periódicos famosos de Londres; resaltando que el modelo de progresión temática lineal podía ser aplicado en textos más simples mientras que el modelo de progresión temática derivada podía ser aplicado en textos expositivos y argumentativos. A través de este estudio el autor concluyó que el enseñar incluso la más mínima noción de progresión temática; podría influir de manera positiva en la producción escrita.

El estudio de la progresión temática aporta a la cohesión y coherencia de un texto en inglés como lengua extranjera puesto que al organizar los elementos constitutivos del texto a nivel de componentes tema-rema habrá mayor jerarquización y concatenación de ideas. El continuo interés por desarrollar una producción escrita más efectiva hace que el estudio de la progresión temática trascienda las fronteras es así que cada vez más se pretende desarrollar las destrezas comunicativas de quienes aprenden inglés como lengua extranjera en distintos lugares del mundo.

Li Jingxia (2013), en su estudio "An Empirical Study on the Application of Theme Theory in the Field of Writing Pedagogy"; exploró el efecto de los modelos de progresión temática en la producción escrita en inglés como lengua extranjera (EFL). El autor condujo un experimento de 10 semanas durante las cuales; la instrucción en escritura académica se desarrolló desde dos perspectivas: La instrucción tradicional aplicada al grupo control, y la instrucción en progresión temática aplicada al grupo experimental. Los hallazgos comprobaron la eficacia de la instrucción en progresión temática en 
la pedagogía de la escritura académica a la vez que se reportaron mejorías en la cohesión y coherencia.

En nuestro contexto académico; la instrucción en progresión temática como estrategia aplicada a la producción escrita de diferentes tipos de texto en inglés como lengua extranjera, es una alternativa nueva que podría contribuir de manera significativa en el proceso enseñanza-aprendizaje. A pesar de que la progresión temática es un tema poco explorado, no se debe restar su importancia en el logro de las propiedades textuales particularmente, la cohesión y coherencia

La cohesión y la coherencia no son las únicas propiedades del texto, sin embargo; ambas son muy esenciales para contribuir a la textualidad. Un texto no puede ser considerado como tal si no hay correspondencia lógica entre sus elementos por esta razón, a través de la progresión temática se busca que los/las estudiantes logren un mejor uso de los recursos cohesivos y un mejor uso de transiciones para comunicar con mayor efectividad sus ideas por medio del lenguaje escrito.

Cabrera \& Beatriz (2012), en su estudio "Comparative Study of Theme Functions in Spanish and English Academic Essays" llevado a cabo en el Salvador; resalta la dificultad de los y las hispano-hablantes al organizar un mensaje y transmitirlo en una lengua extranjera, y cómo el conocimiento de la estructura tema-rema a nivel comparativo y contrastivo (inglés/español) beneficia no sólo a hispanohablantes sino también a anglo-hablantes. El estudio se enfoca en la diferenciación de los elementos tema-rema por medio de un análisis contrastivo para determinar las variantes de tema encontradas en diferentes ensayos escritos en inglés y español para concluir que los ensayos en inglés presentan un mayor número de estructuras temáticas a nivel de cláusulas independientes.

El hecho de que quienes están aprendiendo el inglés como lengua extranjera; desarrollen su habilidad para diferenciar entre los componentes tema-rema en un texto, conlleva a un incremento en su habilidad para recrear estos elementos dentro de un contexto, es decir, cuando los/las estudiantes logran discriminar lo que es tema y lo que es rema, logran establecer diferencias entre la información conocida y la información nueva y es este conocimiento lo que les permite crear asociaciones lógicas para transmitir sus ideas.

La progresión temática también contribuye a la traducción ya que al determinar los elementos tema-rema es posible conocer los patrones estructurales de la información para organizarla de manera efectiva a través del uso correcto los elementos cohesivos (reiteración, sinónimos, conjunciones, conectores, entre otros) lo que conllevará a su vez a una interpretación más coherente. 
Para Kintsch \& Van Dijk (1978), la superestructura hace referencia a la forma que adopta un texto. Esto significa que el contenido de una composición escrita se organiza a través de un esquema, el cual varía de acuerdo al tipo de texto; por ejemplo, el esquema estructural básico de un texto narrativo difiere de aquél que se emplea para un texto argumentativo. Este principio de superestructura es de gran utilidad en la producción escrita de textos en inglés ya que el uso de un esquema facilita la adecuación del lenguaje a diferentes tipos de texto.

Bernárdez (1987), identifica a la lingüística del texto como el estudio científico y lingüístico de las unidades en que efectivamente se produce la comunicación verbal o escrita.

Se conoce como Lingüística del Texto a la disciplina relativamente moderna de carácter interdisciplinario, cuyo objeto es el estudio del texto entendido como un evento de comunicación en distintos planos y sentidos.

Guerrero (1994), señala que esta ciencia tiene por objeto cubrir el vacío que la lingüística oracional dejó tomando en cuenta el cambio que representa la hipótesis de partida según la cual no nos expresamos por medio de frases, sino por medio de textos.

Según Alonso (1997), la escuela de Praga "desarrolló desde una perspectiva funcional un marco conceptual para clasificar y caracterizar estas cadenas informativas" que Danés denominó modelos de progresión temática.

La progresión temática es el mecanismo que permite le fluidez, dosificación y organización de la información dentro de un texto, siendo uno de los fenómenos que más claramente expresa la cohesión textual pues permite el desarrollo de un tema en forma progresiva y la adición de información nueva.

La progresión temática se define, de acuerdo con Suárez (2014) como el mecanismo que organiza y dosifica la información que consta en un texto de manera que se recepte el mensaje con facilidad, esta organización de conceptos clasificados en temas y remas se ajustan a ordenamientos y funciones esquemáticas llamados patrones o modelos de progresión técnica, estos determinan el comportamiento y función de sus elementos y básicamente pueden ser: lineal, constante y derivada, la cual es objeto de este análisis.

Los aportes en progresión temática de Frantisek Danés (1974), nacen después de evidenciar problemas de cohesión textual; de modo que él desarrolló un marco conceptual para determinar cómo la información se distribuye en el texto. De acuerdo a esta premisa Danés establece 3 modelos de progresión temática.

En lo que concierne a los elementos cohesivos, para Anderson (2011), es necesario tener en cuenta algunos mecanismos concretos que serán de gran ayuda si se los usa correctamente como las transiciones, puntuación, 
gramática, antecedente, repetición y sinónimos. El uso de estos recursos dará como resultado la organización lógica

Retomando las palabras de Halliday (1994), el empleo de tematización permite la fluidez de la información por medio de la combinación adecuada de los elementos, tema y rema.

La tematización es el proceso relacionado con la progresión temática y la construcción de textos expositivos. La interrelación tema-rema permite diseñar distintas formas de relacionar la información conocida y la información nueva dentro de la expresión expositiva, pretendiendo combinar dos aspectos que se habían tratado antes, el modelo de progresión temática derivada con el tipo de texto expositivo.

Según Hartley (2008); el lenguaje formal y la estructura gramatical son recursos que definen a la escritura académica como un estilo particular de expresión escrita usado para transmitir las ideas respecto a una disciplina o área específica

Chin, et al (2012 pp V, 32), considera a la escritura académica como un término general que hace referencia a todo trabajo escrito para un propósito de estudio y que además posee un estilo formal, objetivo y lógico, diferente del lenguaje oral o la escritura informal o personal.

Para Irvin (2010), el éxito en la escritura académica está dado por un claro entendimiento de lo que se está haciendo al momento de escribir, para ello, existen varios tipos de escritura académica como reportes, artículos, casos de estudio, revisiones, descripciones, narraciones, resúmenes y ensayos entre otros. Todos ellos obedecen a una tipología textual específica (narrativa, descriptiva, argumentativa, expositiva) creada por T. Van Dijk (1974) en la lingüística del texto.

El presente análisis de los textos escritos en inglés por los/las estudiantes parte desde la perspectiva del texto como producto puesto que se pretende aplicar los principios tema-rema basados en la lingüística textual para lograr textos más coherentes y cohesivos y de este modo retomar su propósito comunicativo a fin de que quien lo escriba logre transmitir sus ideas de manera más efectiva.

\section{METODOLOGÍA}

La investigación fue de tipo cuasi-experimental, en vista de que el grupo experimental y el grupo de control comparten las mismas características, y fueron elegidos de manera intencional. El carácter cuasi experimental de esta investigación hizo posible obtener resultados conducentes a identificar, si el modelo de progresión temática derivada constituyó realmente una estrategia efectiva para el desarrollo de la competencia escrita.

La investigación fue de campo, ya que los datos se obtuvieron directamente de los sujetos investigados y la observación del fenómeno de 
estudio se realizó en su mismo lugar de origen. La investigación es de carácter seccional o transversal ya que los datos fueron obtenidos por única vez en un tiempo límite de 8 semanas.

El estudio fue cuantitativo, ya que los datos obtenidos se sometieron a un análisis estadístico que permitió evidenciar el comportamiento de la variable dependiente después de la aplicación del tratamiento en la fase de intervención, y cualitativo porque fue necesario establecer preguntas y cuestiones que no son medibles en escala numérica

El estudio fue de nivel correlacional, ya que se midió el grado de relación existente entre dos variables; una independiente que es el Modelo de Progresión Temática Derivada y otra dependiente que es la producción escrita de textos expositivos en inglés.

La población estuvo conformada por 64 estudiantes de sexto nivel de inglés del Centro de Idiomas de la ESPOCH, divididos en dos grupos; uno de control correspondiente a los/las estudiantes de sexto nivel paralelo "O" y uno experimental correspondiente al sexto nivel paralelo " $\mathrm{R}$ " ambos, conformados por hombres y mujeres de edades entre 20 y 22 años que asisten a clase durante el período Octubre 2016 - Marzo 2017, y que provienen de las diferentes facultades, escuelas y carreras existentes en la institución.

La fase experimental de este estudio, se llevó a cabo con docentes y estudiantes del Centro de Idiomas de la Escuela Superior Politécnica de Chimborazo. Los/las docentes colaboraron para obtener los datos del grupo control, mientras que los/las estudiantes formaron parte de un grupo experimental y uno de control, los cuales fueron sometidos a pre test y post test.

La experimentación tuvo una duración total de 32 horas clase por semana. Se organizaron los grupos experimental y de control, que fueron evaluados a través de un pre-test conformado por un texto expositivo (ensayo básico) cuyos indicadores se midieron a través de una rúbrica, en la que constan parámetros como la organización, estructura, cohesión y coherencia que son características atribuibles al texto expositivo.

Luego se procedió con una etapa de intervención de forma gradual y sucesiva, que se fundamentaron en los resultados obtenidos del grupo experimental. La etapa de intervención aplicada fue un modelo de progresión temática derivada en las clases de inglés, a fin de evaluar su efecto en la destreza de escritura académica para la producción de textos expositivos en inglés. La secuencia de este proceso obedece a un estudio a nivel de superestructura, que involucra el reconocimiento de los componentes principales del ensayo básico (introducción, desarrollo y conclusión), que se evaluaron a través de una actividad grupal.

Seguidamente, se procedió al estudio del texto a nivel de macro estructura para entender el propósito comunicativo de cada una de las partes 
principales del ensayo; a la vez que se le asignó un número a cada extracto de información que lo compone, para proceder a un análisis micro estructural del texto. Para dicho efecto, se realizaron actividades se utilizaron patrones de textos expositivos de evaluaciones internacionales tipo ensayo tales como el Writing TOEFL IBT (Michael, 2015); estructurados o adaptados bajo el modelo de progresión temática derivada. Se comenzó por el análisis súper estructural y macro estructural del texto tomando en cuenta los componentes del texto expositivo: Introducción, cuerpo, y conclusión.

Posteriormente, se efectuó un análisis de cada uno de los componentes súper estructurales por separado, para proceder a su descomposición micro estructural en oraciones y subsecuentemente determinar los componentes tema-rema para la aplicación del modelo de progresión temática derivada y jerarquizar las diferentes ideas que componen el texto.

Luego se analizó el modelo de progresión temática derivada, tomando como base los elementos tema-rema dentro de la oración analizando su importancia para enlazar las ideas. Dicho análisis se llevó a cabo a través de organizadores gráficos, que sirvieron como medio visual para facilitar la asimilación y comprensión de los diferentes modelos de progresión temática como conocimiento introductorio a modelo de progresión temática derivada, objeto del presente estudio.

La fase de aplicación del modelo de progresión temática derivada, se ejecutó de manera guiada, a través de esquemas básicos que permitieron diferenciar el hiper-tema, (tema general) con sus respectivos subtemas y subremas, para facilitar la estructuración y organización de ideas y así transmitir la información de manera coherente y cohesiva. Al final de cada actividad, se midió el nivel de logro a través de preguntas de reflexión y actividades de retroalimentación sobre el proceso para tener una mejor apreciación del nivel de logro alcanzado por los estudiantes.

Luego de analizar 8 ensayos en etapas sucesivas, el grupo experimental trabajó de forma autónoma en la identificación y uso del modelo de progresión temática derivada, que corresponde a la fase final de la intervención. Las que fueron registrada por cada actividad.

Posterior a la intervención, se evaluó la producción escrita de los estudiantes en el grupo experimental, aplicando un post test con las mismas características del pre test. Los resultados del pre test y del post test fueron tabulados para analizar el efecto de la progresión temática derivada sobre la producción escrita de textos expositivos en inglés.

Se empleó la técnica de la encuesta, para observar el comportamiento de las variables. Para el análisis de información se recurrió a la lectura, al análisis documental, de contenido, sistematización e interpretación de datos, desviación estándar de datos provenientes del grupo control y del grupo experimental después de aplicar el tratamiento. 
- Cada estudiante fue evaluado mediante una tarea a fin de obtener su calificación sobre 20 puntos, tanto para el Pre y Post test en cada grupo.

- La información obtenida fue tabulada a fin de registrar la afectación por cada una de las opciones de los 34 estudiantes de cada grupo.

- Finalmente se presentaron las evaluaciones obtenidas por cada estudiante.

Para validar la efectividad y confiabilidad de los instrumentos de medición fue necesaria la experticia de tres especialistas en el área, 2 Docentes de la Escuela Superior Politécnica de Chimborazo, y un Docente de la Universidad Central del Ecuador, quienes aportaron con su conocimiento y observaciones para la creación de un instrumento capaz de evaluar los indicadores a través del pre test y post test para garantizar la confiabilidad del instrumento y sus resultados alcanzados.

\section{RESULTADOS}

A continuación, los resultados obtenidos mediante la aplicación de las rùbricas de evaluación considerando los componentes para el contexto de la presente investigación

Tabla 1. Frecuencia de las Opciones por cada componente (Pre Test - Grupo Control)

\begin{tabular}{ccccccc}
\hline RÚBRICA & EXCELLENT & GOOD & ACCEPTABLE & REGULAR & POOR & TOTAL \\
\hline Organization & 0 & 2 & 11 & 16 & 5 & 34 \\
Structure & 0 & 1 & 17 & 14 & 2 & 34 \\
Cohesion & 0 & 2 & 11 & 17 & 4 & 34 \\
Coherence & 0 & 0 & 13 & 18 & 3 & 34 \\
\hline
\end{tabular}

Fuente: Resultados de la rubrica

Elaborado por: Logroño Mónica, 2017

Tabla 2. Frecuencias de las opciones por reactivo (Pretest- Grupo experimental)

\begin{tabular}{ccccccc}
\hline RÚBRICA & EXCELLENT & GOOD & ACCEPTABLE & REGULAR & POOR & TOTAL \\
\hline Organization & 1 & 9 & 9 & 11 & 4 & 34 \\
Structure & 2 & 6 & 13 & 11 & 2 & 34 \\
Cohesion & 0 & 4 & 11 & 16 & 3 & 34 \\
Coherence & 0 & 6 & 5 & 16 & 7 & 34 \\
\hline
\end{tabular}

Fuente: Resultados de la rubrica

Elaborado por: Logroño Mónica, 2017

Al comparar el estado inicial del grupo control con el del grupo experimental, se evidencia la ausencia de las propiedades textuales cohesión y coherencia en los textos escritos creados por los/las estudiantes de ambos grupos, salvo en el caso de la organización y estructura; propiedades presentes únicamente en el grupo experimental con una puntuación de excelente únicamente logradas por apenas 1 y 2 estudiantes para cada componente respectivamente. Por otro lado, la coherencia arroja un dato interesante ya que 
en el grupo control conformado por 34 estudiantes apenas 3 presentaron coherencia insuficiente, la cual se evidencia en 7 estudiantes del grupo experimental, es decir que el grupo experimental presenta problemas de coherencia con más frecuencia que el grupo control.

Pre test (Grupo de Control vs Grupo

Experimental)

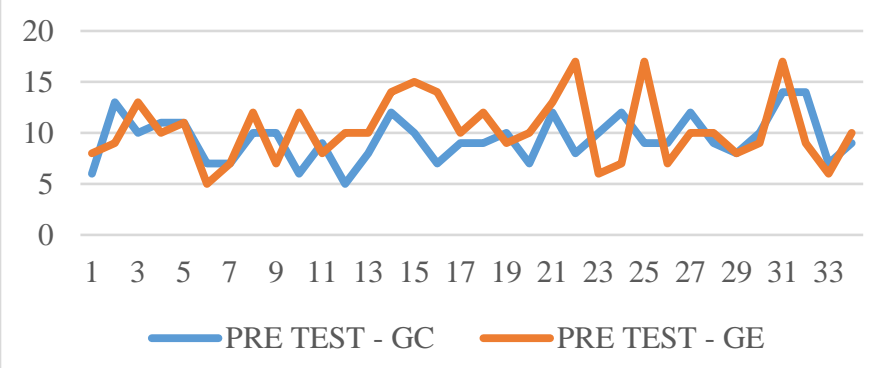

Gráfico 1. Pre test (Grupo control vs Grupo Experimental)

Al comparar los resultados del pre test del grupo control con los del grupo experimental se puede observar que, en el grupo experimental conformado por 34 estudiantes, sobresalen 3 estudiantes que alcanzaron 18 puntos como nota máxima en la evaluación cuyo puntaje óptimo corresponde a 20 puntos. La gráfica indica que los/las estudiantes del grupo experimental superan ligeramente en puntaje a los /las estudiantes del grupo control, ya que pese a haber obtenido mejores calificaciones, éstas ocupan rangos similares.

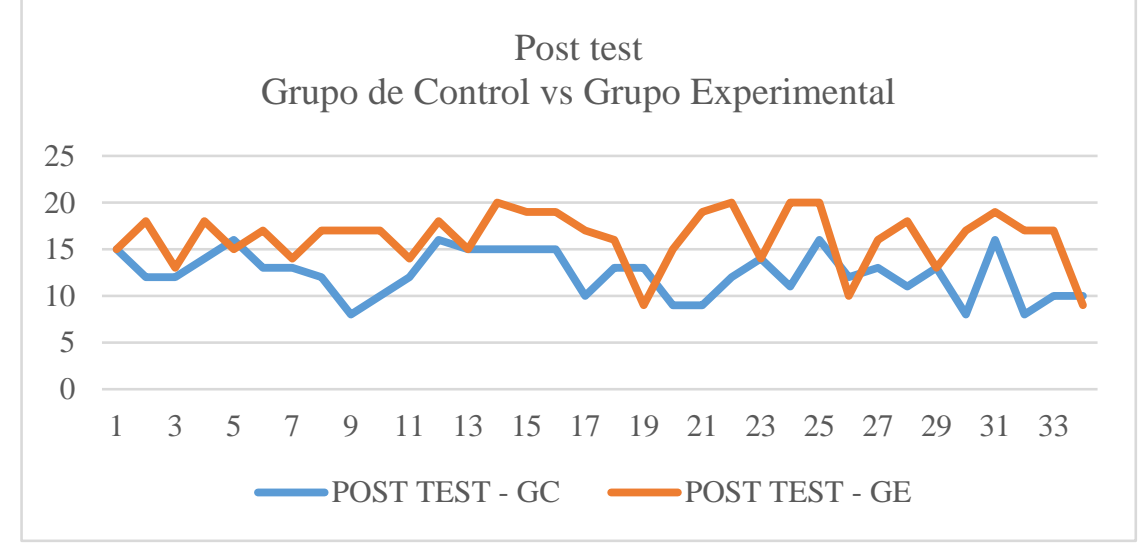

Gráfico 2. Post test (Grupo control vs Grupo Experimental)

A través del gráfico se puede diferenciar que luego del post test los/las estudiantes del grupo experimental obtuvieron mejores calificaciones con respecto a los/las estudiantes del grupo control cuyo promedio reporta 15 puntos con ciertas variaciones que reportan valores inferiores a este rango. El grupo experimental por el contrario reporta un promedio por encima de los 15 
puntos, además, es posible notar que 4 estudiantes obtuvieron un puntaje de 20 puntos que es el óptimo según el instrumento de medición señalado lo cual demuestra que el modelo de progresión temática derivada tiene un efecto significativo sobre la producción de textos expositivos en el Idioma Inglés.

Coeficiente de Correlación

$$
r=\frac{N \sum x y-\sum x \sum y}{\sqrt{\left[N \sum x^{2}-\left(\sum x\right)^{2}\right]\left[N \Sigma y^{2}-(\Sigma y)^{2}\right]}}
$$

Donde:

$\mathrm{r}=$ coeficiente de correlación de Pearson.

Sxy = sumatoria de los productos de ambas variables.

$\mathrm{Sx}=$ sumatoria de los valores de la variable independiente.

Sy $=$ sumatoria de los valores de la variable dependiente.

$\mathrm{Sx}^{2}=$ sumatoria de los valores al cuadrado de la variable independiente.

$\mathrm{Sy}^{2}=$ sumatoria de los valores al cuadrado de la variable dependiente.

$\mathrm{N}=$ tamaño de la muestra en función de parejas.

\begin{tabular}{|r|r|r|r|r|r|}
\hline N. caso & $\mathbf{V I}(\mathbf{X})$ & $\mathbf{V D}(\mathbf{Y})$ & \multicolumn{1}{c|}{$\mathbf{X}^{\mathbf{2}}$} & \multicolumn{1}{c|}{$\mathbf{Y}^{\mathbf{2}}$} & \multicolumn{1}{c|}{$\mathbf{X Y}$} \\
\hline 1 & 10,5 & 11,5 & 110,25 & 132,25 & 120,75 \\
\hline 2 & 12,5 & 13,5 & 156,25 & 182,25 & 168,75 \\
\hline 3 & 11 & 13 & 121 & 169 & 143 \\
\hline 4 & 12,5 & 14 & 156,25 & 196 & 175 \\
\hline 5 & 13,5 & 13 & 182,25 & 169 & 175,5 \\
\hline 6 & 10 & 11 & 100 & 121 & 110 \\
\hline 7 & 10 & 10,5 & 100 & 110,25 & 105 \\
\hline 8 & 11 & 14,5 & 121 & 210,25 & 159,5 \\
\hline 9 & 9 & 12 & 81 & 144 & 108 \\
\hline 10 & 8 & 14,5 & 64 & 210,25 & 116 \\
\hline 11 & 10,5 & 11 & 110,25 & 121 & 115,5 \\
\hline 12 & 10,5 & 14 & 110,25 & 196 & 147 \\
\hline 13 & 11,5 & 12,5 & 132,25 & 156,25 & 143,75 \\
\hline 14 & 13,5 & 17 & 182,25 & 289 & 229,5 \\
\hline 15 & 12,5 & 17 & 156,25 & 289 & 212,5 \\
\hline 16 & 11 & 16,5 & 121 & 272,25 & 181,5 \\
\hline 17 & 9,5 & 13,5 & 90,25 & 182,25 & 128,25 \\
\hline 18 & 11 & 14 & 121 & 196 & 154 \\
\hline 19 & 11,5 & 9 & 132,25 & 81 & 103,5 \\
\hline 20 & 8 & 12,5 & 64 & 156,25 & 100 \\
\hline 21 & 10,5 & 16 & 110,25 & 256 & 168 \\
\hline 22 & 10 & 18,5 & 100 & 342,25 & 185 \\
\hline 23 & 12 & 10 & 144 & 100 & 120 \\
\hline 24 & 11,5 & 13,5 & 132,25 & 182,25 & 155,25 \\
\hline 25 & 12,5 & 18,5 & 156,25 & 342,25 & 231,25 \\
\hline 26 & 10,5 & 8,5 & 110,25 & 72,25 & 89,25 \\
\hline 27 & 12,5 & 13 & 156,25 & 169 & 162,5 \\
\hline 28 & 10 & 14 & 100 & 196 & 140 \\
\hline 29 & 10,5 & 10,5 & 110,25 & 110,25 & 110,25 \\
\hline 30 & 9 & 13 & 81 & 169 & 117 \\
\hline 31 & 15 & 18 & 225 & 324 & 270 \\
\hline & & & & & \\
\hline
\end{tabular}




\begin{tabular}{|r|r|r|r|r|r|}
\hline 32 & 11 & 13 & 121 & 169 & 143 \\
\hline 33 & 8,5 & 11,5 & 72,25 & 132,25 & 97,75 \\
\hline 34 & 9,5 & 9,5 & 90,25 & 90,25 & 90,25 \\
\hline & $\mathbf{3 4 1 , 5}$ & $\mathbf{4 1 8}$ & $\mathbf{3 8 3 7 , 2 5}$ & $\mathbf{5 8 4 6 , 5}$ & $\mathbf{4 6 4 5 , 5}$ \\
\hline & $\sum \mathbf{X}$ & $\sum \mathbf{Y}$ & $\sum \mathbf{X}^{\mathbf{2}}$ & $\sum \mathbf{Y}^{\mathbf{2}}$ & $\sum \mathbf{X Y}$ \\
\hline
\end{tabular}

\begin{tabular}{|c|c|}
\multicolumn{2}{c}{ Coeficiente Correlación 0,37} \\
\hline 0 & INTERPRETACIÓN \\
\hline 0,01 a 0,19 & Correlación nula \\
\hline 0,2 a 0,39 & Correlación positiva muy baja positiva baja \\
\hline 0,4 a 0,69 & Correlación positiva moderada \\
\hline 0,7 a 0,89 & Correlación positiva alta \\
\hline 0,9 a 0,99 & Correlación positiva muy alta \\
\hline 1 & Correlación positiva grande y perfecta \\
\hline
\end{tabular}

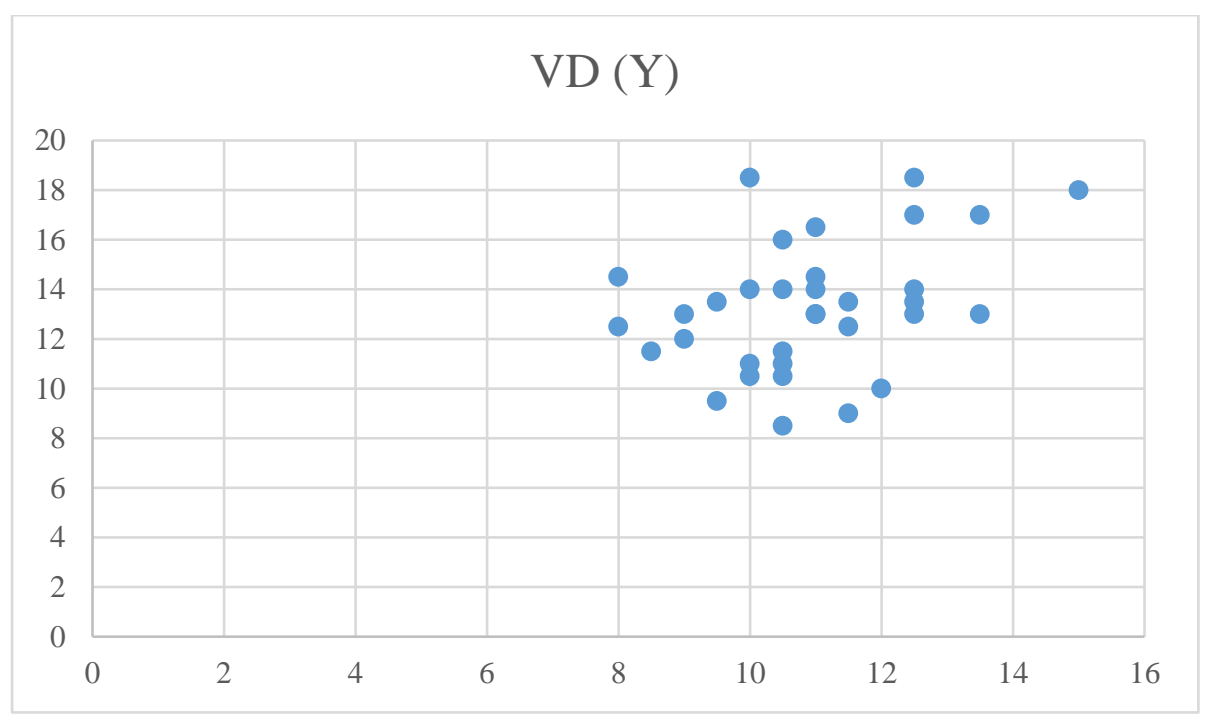

Gráfico3. Dispersión de coeficiente de correlación

Calculando el coeficiente de correlación es de 0,37 y en base a la tabla de interpretación se puede concluir que la Correlación positiva baja

Análisis y prueba de hipótesis

$\mathbf{H}_{1}$ : La aplicación del modelo de progresión temática derivada Sí tiene un efecto significativo sobre la producción de textos expositivos en el Idioma Inglés.

$\mathbf{H}_{0}$ : La aplicación del modelo de progresión temática derivada No tiene un efecto significativo sobre la producción de textos expositivos en el idioma inglés. 


\section{Lenguaje matemático}

\begin{tabular}{|c|c|c|c|c|c|}
\hline \multirow{4}{*}{\multicolumn{2}{|c|}{$\begin{array}{l}\text { PRE TEST } \\
\text { POST TEST }\end{array}$}} & \multicolumn{2}{|c|}{ Media aritmética } & \multicolumn{2}{|c|}{ Desviación estándar(s) } \\
\hline & & \multicolumn{2}{|c|}{9,41176471} & \multicolumn{2}{|r|}{2,22} \\
\hline & & \multicolumn{2}{|c|}{12,3823529} & \multicolumn{2}{|r|}{2,44} \\
\hline & & \multicolumn{2}{|c|}{10,89706} & \multicolumn{2}{|r|}{2,331031} \\
\hline \multicolumn{6}{|c|}{ Grupo experimental } \\
\hline \multicolumn{2}{|c|}{ Rubrica } & \multicolumn{2}{|c|}{ Media aritmética } & \multicolumn{2}{|c|}{ Desviación estándar(s) } \\
\hline \multirow{6}{*}{$\begin{array}{l}\text { PRE TEST } \\
\text { POST TEST }\end{array}$} & \multirow[b]{3}{*}{ Promedio General } & \multicolumn{2}{|c|}{10,35} & \\
\hline & & \multicolumn{2}{|c|}{16,24} & \multirow{2}{*}{\multicolumn{2}{|c|}{$\begin{array}{c}3,16 \\
2,92 \\
\mathbf{3 , 0 4 1 1 4 6}\end{array}$}} \\
\hline & & \multicolumn{2}{|c|}{13,29412} & & \\
\hline & GRUPO & $\mathbf{N}$ & Promedio & \multicolumn{2}{|l|}{ Desv Est. } \\
\hline & Control & 34 & 10,90 & 2,33 & \\
\hline & Experimental & 34 & 13,29 & 3,04 & \\
\hline
\end{tabular}

Determinación de valores críticos y zonas de rechazo.

Mediante el cálculo de la prueba paramétrica $\mathrm{Z}$ se rechaza la hipótesis nula si:

$$
Z_{C}<-Z_{T} \quad Z_{C}<=-1,96 \underset{1,96}{O} \text { Otambién } \quad Z_{C}>Z_{T} \quad Z_{C}>=
$$

Donde $Z_{T}$, es el valor teórico de $\mathrm{Z}$ para un nivel de significación del $5 \%, \alpha=0,05$; es decir que la investigación tendrá un $95 \%$ de confiabilidad; caso contrario se acepta la hipótesis de investigación con una de las dos alternativas.

$$
\begin{aligned}
& \overline{x_{e}}=\mathbf{1 3 , 2 9} \quad \overline{x_{c}}=\mathbf{1 0 , 9 0} \quad \sigma_{\mathrm{e}}=\mathbf{3 , 0 4} \quad \sigma_{\mathrm{c}}=\mathbf{2 , 3 3} \quad n_{e}=34 \\
& n_{c}=34 \\
& Z=\frac{\overline{x_{e}}-\overline{x_{c}}}{\sqrt{\frac{\sigma_{e}^{2}}{n_{e}}+\frac{\sigma_{c}^{2}}{n_{c}}}} \\
& Z_{c}=3,65
\end{aligned}
$$

Toma de decisión estadística.

$\mathrm{Al}$ comparar el valor de $\mathrm{Z}$ calculado y el valor de $\mathrm{Z}$ teórico tenemos

$$
\begin{gathered}
Z_{C}>Z_{T} \\
3,65>1,96
\end{gathered}
$$


Podemos observar, que $Z_{C}=3,65$ está en la zona de rechazo de la hipótesis nula, lo cual nos lleva a rechazar la hipótesis nula $H_{0}: \bar{x}_{e}=\bar{x}_{C} \mathrm{y}$ aceptar la hipótesis de investigación Hi: $\bar{x}_{e} \neq \bar{x}_{C}$ con la alternativa $\mathrm{A}_{1}: \bar{x}_{e}>$ $\bar{x}_{C}$,

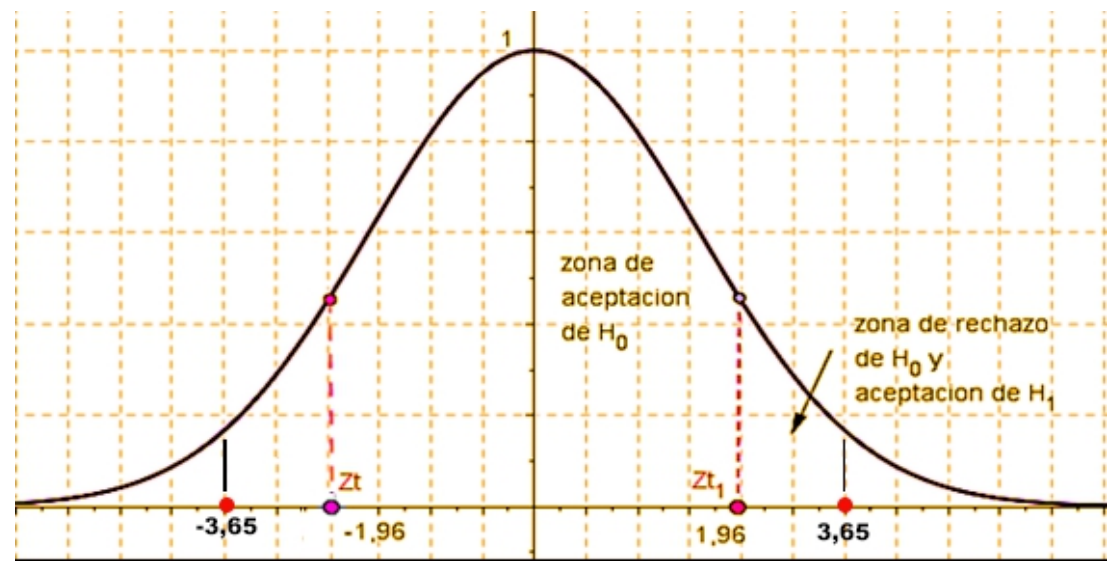

Gráfico 1: Valores de la Z Teórico y $Z$ Calculado

Con un nivel de significación de 0,05 equivalente al $+/-1,96$, se rechaza la hipótesis nula Ho ya que la solución $\mathrm{Zc}=3,65$ se encuentra en el intervalo de la región de rechazo de Ho. En consecuencia, se acepta la hipótesis alternativa H1. Es decir que "La aplicación del modelo de progresión temática derivada tiene un efecto significativo sobre la producción de textos expositivos en el Idioma Inglés."

\section{DISCUSIÓN}

Los datos del pre test indican que tanto el grupo experimental como el de control se encuentran en las mismas condiciones pese a pequeñas diferencias en los componentes evaluados en la producción escrita de textos expositivos en inglés, estas variaciones fluctúan entre 0 y 1 estudiantes en los diferentes niveles de logro por lo los 2 grupos permanecieron dentro de un mismo parámetro académico previo a la aplicación de la estrategia de progresión temática derivada en el grupo experimental.

En cuanto al componente organización, pese a que los datos del post test revelan la ausencia de un nivel pobre por parte del grupo control, el nivel excelente de organización tuvo un incremento del 3\%, lo cual es bastante bajo comparado con el del grupo experimental que demostró un incremento superior al 50\% en el nivel de excelencia siendo éste su nivel predominante; mientras que el nivel predominante del grupo control corresponde al nivel de bueno.

Respecto a este a punto, el error detectado con más frecuencia en ambos grupos fue precisamente la ausencia de un esquema estructural del texto en 
base al cual los/las estudiantes logren desarrollar sus ideas. Para lograr un texto como unidad global primero se debe partir de los principios de súper estructura para garantizar su micro estructura y macro estructura, donde el modelo de progresión temática derivada demostró tener gran utilidad.

El segundo componente hace referencia a la estructura gramatical, cuyos resultados luego del post test reflejan la ausencia de un nivel pobre en estructura, así como la ausencia de un nivel excelente en estructura, además de un ligero incremento en el nivel bueno. El nivel de estructura predominante en los/las estudiantes del grupo control es aceptable. Por otro lado, el nivel de estructura predominante en el grupo experimental se ubica en bueno con un moderado incremento en el nivel de excelencia por lo que la estrategia propuesta contribuye a la estructura gramatical.

Durante la fase experimental, en algunos de los casos, se pudo notar cierto temor al uso de estructuras más complejas (adjective, noun, and adverb clauses) por parte de los/las estudiantes, pese a haberlas estudiado y practicado en sus cursos anteriores, hecho que de alguna manera pudo haber influenciado en los resultados. Un texto para ser considerado como tal debe reunir una estructura lógica adecuada, con el ordenamiento y posicionamiento de la información, lo que concierne a la progresión temática. Los resultados tanto del grupo control como del grupo experimental invitan a la reflexión por parte de los/las docentes en cuanto al uso de estructuras complejas en los niveles superiores.

La cohesión fue el tercer componente analizado. Respecto a la cohesión, el grupo control reporta la ausencia de un nivel excelente al igual que la ausencia de un nivel pobre de cohesión. El nivel predominante de cohesión en la producción escrita de textos expositivos en inglés corresponde a aceptable.

El grupo experimental por su parte, reporta un nivel de cohesión que va de excelente a bueno luego de un periodo de instrucción basado en la aplicación de la progresión temática tomando como principal patrón a seguir, el modelo de progresión temática derivada cuyo aporte fue significativo para incrementar la cohesión textual, ya que en la fase de diagnóstico el grupo experimental reportó ausencia en el nivel excelente mientras que el grupo control mantiene esta ausencia aun después del post test.

Los/las estudiantes del grupo experimental lograron textos más cohesivos, sin embargo, las estructuras gramaticales usadas para tal efecto no siempre correspondieron al nivel esperado ya que como se mencionó anteriormente se reportó un limitado uso de construcciones gramaticales complejas cuyo correcto uso es un requerimiento en la escritura académica en inglés.

En vista de que la cohesión al igual que la coherencia, es una propiedad textual de amplias dimensiones fue necesario afianzar la destreza de los/las estudiantes en el uso de conjunciones y transiciones para permitir el flujo de 
ideas. El aspecto más importante que hizo posible obtener resultados significativos en la cohesión fue la diferenciación de los elementos tema-rema puesto que los/las estudiantes lograron establecer relaciones entre la información nueva y conocida para después jerarquizarla a través del modelo de progresión temática derivada.

Finalmente, se evaluó la coherencia como el último de los componentes ya que es la propiedad textual que le da sentido al texto tanto a nivel local como a nivel global. El grupo control reportó un ligero cambio en la coherencia con una variación de nula a buena, además de la ausencia del nivel pobre y el nivel excelente de coherencia; predominando en este grupo el nivel aceptable con una leve tendencia a regular, mientras que el grupo experimental reportó una coherencia que varía de un nivel bueno con tendencia a excelente.

Al comparar estos resultados es posible determinar el efecto significativo que el modelo de progresión temática derivada aporta a la coherencia del texto expositivo considerando el hecho de que el grupo experimental inicialmente mostraba un nivel pobre de coherencia más alto con respecto al del grupo control. Otro aspecto derivado de los resultados del componente coherencia es que la mayoría de estudiantes del grupo experimental logró una coherencia entre buena y excelente con predominio del nivel bueno

\section{CONCLUSION}

Luego de haber aplicado el modelo de progresión temática derivada; se logró alcanzar una organización a nivel de excelente tanto en el grupo experimental como en el grupo de control.

La diferenciación de los elementos tema rema, es un paso importante en el análisis del texto, ya que se demostró su efectividad en el incremento de la cohesión. El establecer un patrón de ordenamiento de los elementos del texto, permite el flujo de una idea hacia otra, y esto a su vez favorece la mejora de la coherencia en el sentido local y global del texto, atribuyéndole mayor sentido lógico.

La estructura gramatical juega un papel muy importante para establecer un orden lógico. El 17\% del grupo experimental presentó un nivel de estructura excelente; a diferencia del grupo control que reportó ausencia del nivel excelente en ésta característica. Además, el grupo experimental reportó estar un nivel por encima del grupo control, es decir, el 59\% del grupo experimental alcanzó una estructura buena mientras que el 53\% del grupo control logró una estructura aceptable.

La identificación de los elementos tema-rema facilita el proceso de discriminación entre la información conocida y desconocida; sin dejar de lado el rol de los elementos cohesivos tanto a nivel de oración como a nivel de párrafo. Sin embargo, al tratarse de estructuras gramaticales más complejas 
como las de tipo clause; se requiere un mayor monitoreo en el reconocimiento de los temas y remas. El tener en claro cuáles son los componentes, redujo considerablemente el uso de ideas irrelevantes y fuera de contexto.

El modelo de progresión temática derivada representa un recurso de gran utilidad, ya que facilita el proceso de jerarquización y clasificación de información. Permitiendo a los/las estudiantes desarrollar su competencia escrita, y adquirir mayor conciencia de lo que significa escribir un texto expositivo a nivel de súper estructura, macro estructura, y microestructura, tal como lo reporta el 56\% del grupo experimental que logró una organización excelente.

El análisis del texto sirvió para determinar que los/las estudiantes inicialmente mostraron una mayor tendencia al uso de los modelos de progresión temática lineal y constante, los cuales son más representativos de textos de organización más simple. Esto, explica la evasión del uso de estructuras más complejas tipo clause por parte de los/las estudiantes, hecho que se refleja en el 9\% del grupo control; cuya estructura gramatical presentó un nivel regular, mientras que el grupo experimental reportó una reducción de este nivel en un 3\% luego de la aplicación del modelo de progresión temática derivada.

La organización de un texto no se encuentra totalmente basada en un mismo modelo de progresión temática, ya que en un texto pueden darse varios tipos de progresiones. Sin embargo, el modelo de progresión temática derivada predomina en los textos expositivos.

El modelo de progresión temática derivada contribuye a la cohesión y coherencia del texto. Es así, que el 9\% del grupo experimental que reportó un nivel excelente de cohesión, se incrementó a 50\% luego de la aplicación del modelo de Progresión Temática Derivada. Por otro lado, en cuanto a la coherencia; los resultados del grupo experimental reportan un aumento del $0 \%$ al $20 \%$ en el nivel de coherencia excelente, por lo que se debe tomar en cuenta que la coherencia es una propiedad textual que se valora a nivel global y local, y requiere mayor práctica, sobre todo al relacionarla con el componente cohesión.

La cohesión y coherencia son dos propiedades del texto que se encuentran estrechamente relacionadas, pero que difieren entre sí, tal como lo muestran las variaciones de $50 \%$ y $20 \%$ respectivamente. Un texto puede ser cohesivo, pero no coherente o un texto puede ser coherente pero no cohesivo.

\section{References:}

1. Alonso, M. I. (1997). Un estudio de la progresión temática en las composiciones escritas de los alumnos de. ASELE, 1-10.

2. Bernárdez, E. (1987). Lingüistica del Texto. Barcelona: Arco libros. 
3. Cabrera, P. de, \& Beatriz, L. (2015). Comparative study of theme functions in spanish and english academic essays. Recuperado a partir de http://www.redicces.org.sv:80/jspui/handle/10972/2205

4. Chin, P., Koizumi, Y., Reid, S., wray, S., \& Yamazaki, Y. (2012). Academic writing Skills, student book 1. Melbourne: Cambridge University press.

5. Daneš, F. (1974). Papers on Functional Sentence Perspective. Academia.

6. Guerrero, G. (1994). La lingûística del texto y la pragmática lingûística. ELUA, 443 - 446.

7. Halliday, M. A. K. (1994). An Introduction to Functional Grammar. London: Edward Arnold

8. Hartley, J. (2008). Academic Writing and Publishing. New York: Routledge.

9. Hawes, T. (2014). Thematic Progressions in the Wrting of Students and Professionals. Elsevier, 93-100.

10. Liu Jingxia. (2013). An Empirical Study on the Applicat ion of Theme Theory in the Field of Writing Pedagogy, 6. Recuperado a partir de https://files.eric.ed.gov/fulltext/EJ1077030.pdf

11. Irvin, L. (2010). What's Academic writing? Readings on Writing, Volume 1, 3-5.

12. Kintsch, W., \& van Dijk, T. A. (1978). Toward a model of text comprehension and production. Psychological Review, 85(5), 363394. https://doi.org/10.1037/0033-295X.85.5.363

13. Ping, A. L. (2007). Developing the message: Thematic Progression and Student writing. The Journal of Asia, 93-117.Daneš, F. (1974). Papers on Functional Sentence Perspective. Academia.

14. EJ1077030.pdf. (s. f.). Recuperado a partir de https://files.eric.ed.gov/fulltext/EJ1077030.pdf

15. Liu Jingxia. (2013). An Empirical Study on the Applicat ion of Theme Theory in the Field of Writing Pedagogy, 6. Recuperado a partir de https://files.eric.ed.gov/fulltext/EJ1077030.pdf 